\title{
ON SPACES OF THE SAME STRONG $n$-TYPE
}

\author{
YVES FÈLIX AND JEAN-CLAUDE THOMAS
}

(communicated by Lionel Schwartz)

\begin{abstract}
Let $X$ be a connected CW complex and $[X]$ be its homotopy type. As usual, $\operatorname{SNT}(X)$ denotes the pointed set of homotopy types of CW complexes $Y$ such that their $n^{\text {th }}$-Postnikov approximations $X^{(n)}$ and $Y^{(n)}$ are homotopy equivalent for all $n$. In this paper we study a particularly interesting subset of $\operatorname{SNT}(X)$, denoted $\operatorname{SNT}_{\pi}(X)$, of strong $n$ type; the $n^{t h}$-Postnikov approximations $X^{(n)}$ and $Y^{(n)}$ are homotopy equivalent by homotopy equivalences satisfying an extra condition at the level of homotopy groups. First, we construct a CW complex $X$ such that $\operatorname{SNT}_{\pi}(X) \neq\{[X]\}$ and we establishe a connection between the pointed set $\mathrm{SNT}_{\pi}(X)$ and sub-groups of homotopy classes of selfequivalences via a certain $\lim ^{1}$ set. Secondly, we prove a conjecture of Arkowitz and Maruyama concerning subgroups of the group of self equivalences of a finite $\mathrm{CW}$ complex and we use this result to establish a characterization of simply connected CW complexes with finite dimensional rational cohomology such that $\operatorname{SNT}_{\pi}(X)=\{[X]\}$.
\end{abstract}

\section{Introduction}

Let $X$ be a connected $\mathrm{CW}$ complex and $[X]$ be its homotopy type. As usual, $\operatorname{SNT}(X)$ denotes the pointed set of homotopy types of CW complexes $Y$ such that their $n^{\text {th }}$-Postnikov approximations $X^{(n)}$ and $Y^{(n)}$ are homotopy equivalent (then $X$ and $Y$ have the same $n$ type for all $n \geqslant 1$ ).

The first example of a space $X$ with $\operatorname{SNT}(X) \neq\{[X]\}$ has been given by J.F. Adams in 1957 ([1]). In 1966 Brayton Gray, ([7]), found one with finite type. In general, the determination of spaces $X$ such that $\operatorname{SNT}(X)$ is a singleton remains an open problem. For instance, in $([\mathbf{1 2}])$, C.A. McGibbon and J. Möller conjecture that $\operatorname{SNT}(\Omega X)=\{[\Omega X]\}$ for a simply connected finite CW complex $X$ and prove this conjecture when $X$ is an $\mathrm{H}_{0}$-space, and in fact using, [13], their proof works as well in order to prove the conjecture for any rational elliptic space, $[6]$.

In this paper we consider a particular subset of $\operatorname{SNT}(X)$ denoted $\operatorname{SNT}_{\pi}(X)$ that we now describe. Denote by $P_{X}$ the set of pairs $\left(Y,\left(f_{n}\right)_{n \geqslant 1}\right)$, where $f_{n}: Y^{(n)} \rightarrow X^{(n)}$ is a homotopy equivalence and such that the following diagrams commute for $n \geqslant 2$.

$$
\begin{array}{ccc}
\pi_{*}\left(Y^{(n)}\right) & \stackrel{\pi_{*}\left(f_{n}\right)}{\longrightarrow} & \pi_{*}\left(X^{(n)}\right) \\
\downarrow \pi_{*}\left(p_{Y}^{(n)}\right) & & \downarrow \pi_{*}\left(p_{X}^{(n)}\right) \\
\pi_{*}\left(Y^{(n-1)}\right) & \stackrel{\pi_{*}\left(f_{n-1}\right)}{\longrightarrow} & \pi_{*}\left(X^{(n-1)}\right)
\end{array}
$$

The first author was partially supported by a CNRS research position held in Angers and the second by UPRESA CNRS 60093.

Both authors were partially supported by a CNRS-FNRS-CGRI Tournesol agreement

Received 28 September 1999, revised 18 December 1999; published on 27 December 1999.

1991 Mathematics Subject Classification: 55P10, 55P15, 18G55

Key words and phrases: Derived functor, Postnikov tower, Self-equivalences, $\lim ^{1}$, Phantom map

(C) 1999, Yves Fèlix and Jean-Claude Thomas. Permission to copy for private use granted. 
We put $\operatorname{SNT}_{\pi}(X)=\left\{[Y] \mid\left(Y,\left(f_{n}\right)\right) \in P_{X}\right\}$.

When $[Y]$ belongs to $\operatorname{SNT}_{\pi}(X)$, we say that $X$ and $Y$ have the same strong $n$ type.

It follows from the works of Adams ([1]), Wilkerson ([18]), McGibbon and Möller ([12], [10]) that

$$
\operatorname{SNT}(X)=\operatorname{SNT}_{\pi}(X)=\{[X]\}
$$

in each of the following cases

(a) if $\pi_{i}(X)$ is a finite group for all $i>0$;

(b) if $X$ is a simply connected finite type rational space;

(c) if $X$ is a simply connected, $\mathbb{Q}$-finite type, $H_{0}$-space such that the natural map $\operatorname{Aut}(X) \rightarrow$ Aut $H^{\leqslant n}(X ; \mathbb{Z})$ has a finite cokernel for $n \geqslant 1$. Here Aut $H^{\leqslant n}(X ; \mathbb{Z})$ denotes the group of ring homomorphisms.

Example 1. $\left.\operatorname{SNT}_{\pi}(X) \neq\{[X]\}\right)$ when $X$ is the 0-localization of the CW complex

$$
Z=\left(S_{a}^{2} \times S_{b}^{2} \times \bigvee_{p \geqslant 1} S^{6 p}\right) \vee\left(\bigvee_{n \geqslant 1} S_{n}^{4}\right) \cup\left(\gamma_{n, p}\right) \bigcup_{\substack{p \geqslant 1 \\ n>p}} e_{n}^{4+6 p},
$$

where $\gamma_{n, p}$ is the Whitehead bracket $\left[S_{n}^{4}, S^{6 p}\right]$. Observe that $X$ is not of finite type (see $(b)$ above !). More precisely we prove the following equality (Theorem 4 below)

$$
\operatorname{SNT}_{\pi}(X) \cong \prod_{n \geqslant 1} \mathbb{Q} / \bigoplus_{n \geqslant 1} \mathbb{Q}
$$

Our main result establishes a connection between the pointed set $\operatorname{SNT}_{\pi}(X)$ and sub-groups of homotopy classes of self-equivalences via a certain $\lim ^{1}$ set. We will state the precise results with its first consequences after some definitions.

If $X$ is a based topological space, Aut $(X)$ denotes the group of homotopy classes of self homotopy equivalences of $X$, and $\operatorname{Aut}_{\pi}(X)$ the subgroup of homotopy classes which induce the identity on the homotopy groups of $X$. A tower of groups

$$
\cdots \rightarrow \text { Aut } X^{(n)} \stackrel{q_{n}}{\rightarrow} \text { Aut } X^{(n-1)} \rightarrow \cdots \rightarrow \text { Aut } X^{(1)},
$$

is defined by the homomorphisms $q_{n}([f])=\left[f^{(n-1)}\right]$ where $f^{(n-1)}$ denotes the map induced up to homotopy by $f$ :

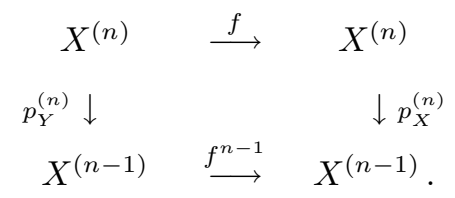

In 1975, C. Wilkerson ([18]) establishes the existence of a natural pointed set bijection

$$
\theta^{X}: \operatorname{SNT}(X) \rightarrow \lim _{\leftarrow}^{1} \text { Aut } X^{(n)} .
$$

where $\lim ^{1} G_{n}$ is defined for a tower of groups

$$
\cdots \rightarrow G_{n} \stackrel{q_{n}}{\rightarrow} G_{n-1} \rightarrow \cdots \stackrel{q_{2}}{\rightarrow} G_{1},
$$

as the orbit set for the action of the group $\prod_{n} G_{n}$ acting on the set $\prod_{n} G_{n}$ by

$$
\left(\gamma_{n}\right) \cdot\left(\alpha_{n}\right)=\left(\gamma_{n} \alpha_{n} q_{n+1}\left(\gamma_{n+1}\right)^{-1}\right) \text {. }
$$


The sequence of injections $\operatorname{Aut}_{\pi} X^{(n)} \rightarrow$ Aut $X^{(n)}$ induces a natural map

$$
j_{X}: \lim _{\leftarrow}^{1} \operatorname{Aut}_{\pi} X^{(n)} \rightarrow \lim _{\leftarrow}^{1} \text { Aut } X^{(n)} .
$$

Theorem 1. Let $X$ be a simply connected $C W$ complex. Then there is a pointed set bijection $\operatorname{SNT}_{\pi}(X) \rightarrow$ Image $j_{X}$.

Example 2. Assume $X$ is a simply connected finite type CW-complex that has the rational homotopy type of a bouquet of spheres. By ([12], Lemma 1), the groups $\operatorname{Aut}_{\pi} X^{(n)}$ are finite, so that $\lim _{\leftarrow}^{1} \operatorname{Aut}_{\pi} X^{(n)}=\{*\}$ and $\operatorname{SNT}_{\pi}(X)=\{[X]\}$, see $[\mathbf{1 2}]$.

Example 3. Denote by $f: \mathbb{C} P^{\infty} \rightarrow S^{3}$ a phantom map and by $H: S^{3} \rightarrow S^{2}$ the Hopf map. The homotopy cofibre $Z$, of $H \circ f$, and the space $S^{2} \vee \Sigma \mathbb{C} P^{\infty}$ have the same $n$ type for all $n$. D. Stanley $([\mathbf{1 6}])$ has proved that the Lusternik-Schnirelmann category of $Z$ is two whereas the category of $S^{2} \vee \Sigma \mathbb{C} P^{\infty}$ is one. Therefore the spaces $Z$ and $S^{2} \vee \Sigma \mathbb{C} P^{\infty}$ do not have the same homotopy type and thus not the same strong $n$ type because they are rational suspensions.

Theorem 2. Let $X$ be a simply connected finite type $C W$ complex with finite dimensional rational cohomology, then $S_{N} T_{\pi}(X)=\{[X]\}$ if and only if for some integer $N$, the morphism $\operatorname{Aut}_{\pi}(X) \rightarrow \operatorname{Aut}_{\pi}\left(X^{(N)}\right)$ has a finite cokernel.

\section{The six term exact sequence}

Let $X$ be a simply connected finite type CW complex. Denote by $G_{n}$ the image of the natural morphism Aut $X^{(n)} \rightarrow \operatorname{Aut} \pi_{\leqslant n}(X)$. Then the short exact sequence of towers

$$
1 \rightarrow \operatorname{Aut}_{\pi} X^{(n)} \rightarrow \operatorname{Aut} X^{(n)} \rightarrow G_{n} \rightarrow 1
$$

induces a 6 -term exact sequence, $[8]$ :

$$
\begin{aligned}
& 1 \rightarrow \lim _{\leftarrow} \operatorname{Aut}_{\pi} X^{(n)} \rightarrow \lim _{\leftarrow} \operatorname{Aut}^{(n)} \rightarrow \lim _{\leftarrow} G_{n} \stackrel{\delta}{\rightarrow} \lim _{\leftarrow}{ }^{1} \operatorname{Aut}_{\pi} X^{(n)} \stackrel{j_{X}}{\rightarrow} \\
& \stackrel{j_{X}}{\rightarrow} \lim _{\leftarrow}{ }^{1} \operatorname{Aut} X^{(n)} \rightarrow \lim _{\leftarrow}^{1} G_{n} \rightarrow *
\end{aligned}
$$

The image of $j_{X}$ is, by Theorem 1 , in bijection with the subset $\operatorname{SNT}_{\pi}(X)$ of $S N T(X)$ so that we obtain a 6 -term exact sequence

$$
1 \rightarrow \operatorname{Aut}_{\pi} X \rightarrow \operatorname{Aut} X \rightarrow \lim _{\leftarrow} G_{n} \stackrel{\delta}{\rightarrow} \lim _{\leftarrow}{ }^{1} \operatorname{Aut}_{\pi} X^{(n)} \stackrel{j_{X}}{\rightarrow} S N T(X) \rightarrow \lim _{\leftarrow}^{1} G_{n} \rightarrow * .
$$

In particular, in the case $\mathrm{SNT}_{\pi}(X)=\{[X]\}$ we have a bijection

$$
\operatorname{SNT}(X) \stackrel{\cong}{\longrightarrow} \lim _{\leftarrow}^{1} G_{n} .
$$

This 6-term exact sequence provides us with anaother description of the set $\mathrm{SNT}_{\pi} X$. The group $\lim G_{n}$ is the subgroup of Aut $\pi_{*}(X)$ consisting of those automorphisms $\varphi$ such that, for each $n \geqslant 1$, the restriction of $\varphi$ to $\pi_{\leqslant n}$ can be realized by some automorphism $\alpha_{n} \in$ Aut $X^{(n)}$. The connecting map $\delta$ associates to $\varphi$ the class of the element $\left(\alpha_{n}\left(\alpha_{n+1}\right)^{-1}\right)_{n \geqslant 1}$ in $\lim ^{1} \operatorname{Aut}_{\pi} X^{(n)}$.

The group $\lim G_{n}$ acts on the set $\lim ^{1} \operatorname{Aut}_{\pi} X^{(n)}$ in the following way : Let $\varphi$ be an element in $\lim G_{n}$ whose restriction to $\pi_{\leqslant n}$ is realized by an automorphism $\alpha_{n} \in \operatorname{Aut} X^{(n)}$, and let $\beta=\left[\left(\beta_{n}\right)\right] \in \lim _{\leftarrow}^{1} \operatorname{Aut}_{\pi} X^{(n)}$, then

$$
(\varphi \cdot \beta)_{n}=\alpha_{n} \beta_{n}\left(\alpha_{n+1}\right)^{-1} .
$$


Therefore we deduce:

Proposition 1. The set $\operatorname{SNT}_{\pi}(X)$ is the orbit space of $\lim _{\leftarrow}^{1} \operatorname{Aut}_{\pi} X^{(n)}$ for this action.

Let us precise here another interesting fact concerning the above 6-term exact sequence. Let aut $_{\pi} X$ denote the monoid of self equivalences which induce the identity map at the level of homotopy groups and recall that $\pi_{0}\left(\operatorname{aut}_{\pi} X\right)=\operatorname{Aut}_{\pi} X$. From the work of Bousfield and Kan $([\mathbf{4}])$, we have a diagram of short exact sequences

$$
\begin{aligned}
& 1 \rightarrow \lim _{\leftarrow}^{1} \pi_{1}\left(\text { aut }{ }_{\pi} X^{(n)}\right) \quad \rightarrow \quad \text { aut }{ }_{\pi} X \stackrel{\rho_{1}}{\rightarrow} \quad \underset{\leftarrow}{\lim } \text { Aut }{ }_{\pi} X^{(n)} \rightarrow 1
\end{aligned}
$$

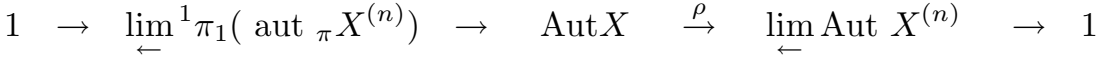

Moreover the kernel of $\rho$ is the subgroup of automorphisms $\varphi$ whose restrictions to $X^{(n)}$ are homotopic to the identity for each $n \geqslant 1$. This subgroup of $\operatorname{Aut}_{\pi} X$ is called the group of weak identities of $X$. Observe that if $X$ is an $H$-space, the set of homotopy classes of phantom maps from $X$ into $X, \operatorname{Ph}(X, X)$, is a group, and that the map $\theta: \operatorname{Ph}(X, X) \rightarrow \operatorname{Ker} \rho$ defined by $\theta(f)=i d+f$ is a group isomorphism $([\mathbf{1 5}])$.

There is another interesting subgroup of $\operatorname{Aut}_{\pi} X^{(n)}$, the subgroup $\operatorname{Aut}_{\Omega} X^{(n)}$ formed by the automorphisms $\varphi$ such that $\Omega \varphi$ is homotopic to the identity. By $([\mathbf{5}])$, the maps $\operatorname{Aut}_{\Omega} X^{(n)} \rightarrow$ Aut $_{\pi} X^{(n)}$ are injections of finitely generated nilpotent groups. Since these injections become isomorphisms after Malcev completion, the quotients $H_{n}=\operatorname{Aut}_{\pi} X^{(n)} / \operatorname{Aut}_{\Omega} X^{(n)}$ are finite groups. Therefore $\lim ^{1} H_{n}=0$, and we have a surjection

$$
\lim _{\leftarrow}^{1} \operatorname{Aut}_{\Omega} X^{(n)} \rightarrow \lim _{\leftarrow}^{1} \operatorname{Aut}_{\pi} X^{(n)}
$$

This shows that

\section{Proposition 2.}

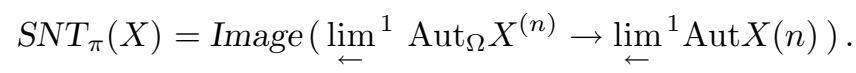

In other words, the set $\mathrm{SNT}_{\pi}(X)$ can also be described as the subset of $S N T(X)$ formed by the spaces $Y$ for which there exist maps $f_{n}: Y^{(n)} \rightarrow X^{(n)}$ such that the following diagrams commute for $n \geqslant 2$.

$$
\begin{array}{ccc}
\Omega Y^{(n)} & \stackrel{\Omega f_{n}}{\longrightarrow} & \Omega X^{(n)} \\
\downarrow \Omega p_{Y}^{(n)} & & \downarrow \Omega p_{X}^{(n)} \\
\Omega Y^{(n-1)} & \stackrel{\Omega f_{n-1}}{\longrightarrow} & \Omega X^{(n-1)}
\end{array}
$$

\section{Proof of Theorem 1}

Recall that $P_{X}$ denotes the set of pairs $\left(Y,\left(f_{n}\right)_{n \geqslant 1}\right)$, where $f_{n}: Y^{(n)} \rightarrow X^{(n)}$ is an homotopy equivalence and such that the following diagrams commute

$$
\begin{array}{ccc}
\pi_{*}\left(Y^{(n)}\right) & \stackrel{\pi_{*}\left(f_{n}\right)}{\longrightarrow} & \pi_{*}\left(X^{(n)}\right) \\
\downarrow \pi_{*}\left(p_{Y}^{(n)}\right) & & \downarrow \pi_{*}\left(p_{X}^{(n)}\right) \\
\pi_{*}\left(Y^{(n-1)}\right) & \stackrel{\pi_{*}\left(f_{n-1}\right)}{\longrightarrow} & \pi_{*}\left(X^{(n-1)}\right)
\end{array}
$$


We introduce an equivalence relation on the set $P_{X}$. Two pairs $\left(Y,\left(f_{n}\right)\right)$ and $\left(Z,\left(g_{n}\right)\right)$ in $P_{X}$ are equivalent if there is an homotopy equivalence $\varphi: Z \rightarrow Y$ such that the maps $f_{n} \circ \varphi \circ g_{n}^{-1}$ belongs to $\operatorname{Aut}_{\pi}\left(X^{(n)}\right)$ for all $n$. For instance, for any homotopy equivalence $m: Y \rightarrow Z$ and each element $\left(Z,\left(g_{n}\right)\right)$, the elements $\left(Y,\left(g_{n} \circ m\right)\right)$ and $\left(Z,\left(g_{n}\right)\right)$ are equivalent. We denote by $E_{X}$ the quotient set $P_{X} / \sim$. To each pair $\left(Y,\left(f_{n}\right)\right) \in P_{X}$ we associate the homotopy type of $Y$ into $\operatorname{SNT}_{\pi}(X)$. We obtain in this way a well defined map $\rho: E_{X} \rightarrow \operatorname{SNT}_{\pi}(X)$.

The following proposition implies then clearly Theorem 1.

Proposition 3. Let $X$ be a simply connected $C W$ complex. There is a natural pointed set bijection $\theta_{1}^{X}: E_{X} \rightarrow \lim ^{1} \operatorname{Aut}_{\pi} X^{(n)}$. Moreover, the bijections $\theta^{X}$ and $\theta_{1}^{X}$ fit together in a commutative diagram

$$
\begin{array}{ccccc}
\lim _{\leftarrow}^{1} \operatorname{Aut}_{\pi} X^{(n)} & \longrightarrow & \text { Image } j_{X} & \hookrightarrow & \underset{\leftarrow}{\lim }{ }^{1} \operatorname{Aut} X^{(n)} \\
\uparrow \theta_{1}^{X} & & \uparrow & & \uparrow \theta^{X} \\
E_{X} & \stackrel{\rho}{\longrightarrow} & \operatorname{SNT}_{\pi}(X) & \hookrightarrow & \operatorname{SNT}(X) .
\end{array}
$$

Proof. Since the simplicial Postnikov decomposition is functorial, we consider Kan complexes instead of CW complexes. The $n^{t h}$-Postnikov complex of a simplicial complex $X$ is the quotient $X^{(n)}=X / \sim_{n}$ where $x \sim_{n} y$ if the corresponding faces of $x$ and $y$ of dimensions less than or equal to $n$ are equal. The quotient $X^{(n)}$ is a Kan complex, and the natural projections

$$
p_{X}^{(n)}: X^{(n)} \rightarrow X^{(n-1)}
$$

are Kan fibrations with Eilenberg-MacLane complexes $K\left(\pi_{n}(X), n\right)$ as fibres.

We suppose that $X$ is a minimal Kan complex. Therefore each $X^{(n)}$ is also minimal. As usual, Aut $X^{(n)}$ denotes the group of simplicial isomorphisms and $\operatorname{Aut}_{\pi} X^{(n)}$ denotes the subgroup of automorphisms inducing the identity map on the homotopy groups.

If $\alpha_{n} \in \operatorname{Aut} X^{(n)}$, the composite $\alpha_{n} \circ p^{(n+1)}$ is again a Kan fibration and a Postnikov section. Let $\bar{\alpha}=\left(\alpha_{0}, \cdots\right)$ denotes the sequence of the $\alpha_{n}$ in $\prod_{n} \operatorname{Aut}_{\pi}\left(X^{(n)}\right)$, and define

$$
X_{\bar{\alpha}}=\lim _{\leftarrow}\left(X^{(n)}, \alpha_{n-1} \circ p^{(n)}\right) .
$$

Then $X_{\bar{\alpha}}$ is again a minimal Kan complex and the commutativity of the square

$$
\begin{array}{ccl}
X^{(n)} & = & X^{(n)} \\
p_{X}^{(n)} \downarrow & & \downarrow^{\alpha_{n-1} \circ p^{(n)}} \\
X^{(n-1)} & \stackrel{\alpha_{n-1}}{\longrightarrow} & X^{(n-1)}
\end{array}
$$

shows that for each $n$ there are homotopy equivalences $f_{n}^{\bar{\alpha}}: X^{(n)} \rightarrow X_{\bar{\alpha}}^{(n)}$ such that the following diagram commutes

$$
\begin{array}{ccc}
\pi_{*}\left(X^{(n)}\right) & \stackrel{\pi_{*}\left(f_{n}^{\bar{\alpha}}\right)}{\longrightarrow} & \pi_{*}\left(X_{\bar{\alpha}}^{(n)}\right) \\
\pi_{*}\left(p_{X}^{(n)}\right) \downarrow & & \downarrow \pi_{*}\left(\alpha_{n-1} \circ p^{(n)}\right) \\
\pi_{*}\left(X^{(n-1)}\right) & \stackrel{\pi_{*}\left(\alpha_{n-1}\right)}{\longrightarrow} & \pi_{*}\left(X^{(n-1)}\right) .
\end{array}
$$

In particular, $\left(X_{\bar{\alpha}},\left(f_{n}^{\bar{\alpha}}\right)\right)$ defines an element in $P_{X}$ and in $E_{X}$.

Suppose that $\bar{\alpha}$ and $\bar{\beta}$ define the same element in $\lim ^{1} \operatorname{Aut}_{\pi} X^{(n)}$. Then there is a sequence $\bar{\gamma}=\left(\gamma_{n}\right) \in \prod_{n}$ Aut $_{\pi} X^{(n)}$ such that $\alpha_{n}=\gamma_{n} \circ \beta_{n} \circ\left(q_{n+1}\left(\gamma_{n+1}\right)\right)^{-1}$. We then have maps 
$\gamma_{n}: X_{\bar{\beta}}^{(n)} \longrightarrow X_{\bar{\alpha}}^{(n)}$ such that the following diagrams commute up to homotopy

$$
\begin{array}{rll}
X^{(n)} & \stackrel{\gamma_{n}}{\longrightarrow} & X^{(n)} \\
\beta_{n-1} \circ p_{X}^{n} \downarrow & & \downarrow \alpha_{n-1} \circ p_{X}^{n} \\
X^{(n-1)} & \stackrel{\gamma_{n-1}}{\longrightarrow} & \left.X^{(n-1}\right)
\end{array}
$$

and thus the homotopy class $\left[\left(f_{n}^{\bar{\alpha}}\right)^{-1} \circ \gamma_{n} \circ f_{n}^{\bar{\beta}}\right]$ belongs to $\operatorname{Aut}_{\pi} X^{(n)}$. Here, if $f$ is a homotopy equivalence, then $f^{-1}$ means any representative for the class $[f]$. The correspondance $\bar{\alpha} \rightarrow$ $\left(X_{\bar{\alpha}},\left(f_{n}^{\bar{\alpha}}\right)\right)$ induces therefore a well defined map

$$
\Phi: \lim _{\leftarrow}^{1} \operatorname{Aut}_{\pi} X^{(n)} \rightarrow E_{X}
$$

Which respects base points.

In order to show that $\Phi$ is a bijection we construct the inverse map. The map

$$
\theta: P_{X} \rightarrow \lim _{\leftarrow}^{1} \operatorname{Aut}_{\pi} X^{(n)}, \quad \theta\left(Y,\left(f_{n}\right)\right)=\left(\left[f_{n} \circ\left(f_{n+1}^{(n)}\right)^{-1}\right]\right)
$$

factors to give a map $\theta_{1}^{X}: E_{X} \rightarrow \lim ^{1} \operatorname{Aut}_{\pi} X^{(n)}$. Indeed, suppose that $\left(Y,\left(f_{n}\right)\right)$ and $\left(Z,\left(g_{n}\right)\right)$ are equivalent, then there exists a map $\varphi$ such that, for each integer $n \geqslant 1$, the class $\alpha_{n}=$ $\left[g_{n} \circ \varphi^{(n)} \circ f_{n}^{-1}\right] \in \operatorname{Aut}_{\pi} X^{(n)}$. This shows that

$$
\left(\alpha_{n}\right) \cdot\left[f_{n} \circ\left(f_{n+1}^{(n)}\right)^{-1}\right]=\left[g_{n} \circ\left(g_{n+1}^{(n)}\right)^{-1}\right] .
$$

The relation $\Phi \circ \theta_{1}^{X}=i d$ results from the commutativity, up to homotopy, of the diagram

$$
\begin{array}{ccl}
Y^{(n)} & \stackrel{f_{n}}{\longrightarrow} & X^{(n)} \\
p_{Y}^{n} \downarrow & & \downarrow f_{n-1} \circ\left(f_{n}^{n-1}\right)^{-1} p_{X}^{n} \\
Y^{(n-1)} & \stackrel{f_{n}^{(n-1)}}{\longrightarrow} & X^{(n-1)}
\end{array}
$$

The relation $\theta_{1}^{X} \circ \Phi=i d$ is trivially satified.

\section{A conjecture of Arkowitz and Maruyama}

For a simply connected CW complex $Z$ we denote by $\operatorname{Aut}_{\#}^{n}(Z), n \geqslant 2$, the kernel of the natural morphism $\operatorname{Aut}(Z) \rightarrow$ Aut $\pi_{\leqslant n}(Z)$. In $([\mathbf{3}])$ Arkowitz and Maruyama conjecture that for a finite simply connected CW complex there is an integer $N$ such that the restriction map $\mathrm{Aut}_{\pi} \rightarrow \mathrm{Aut}_{\#}^{N} Z$ is an isomorphism. Here we prove the conjecture as a corollary of its rational version.

Theorem 3. Let $Z$ be a simply connected finite type $C W$ complex and let $Z_{0}$ its rationalization. Suppose there $H^{>M}(Z ; \mathbb{Q})=0$ for some $M$, then there is some integer $N$ such that the restriction map

$$
\operatorname{Aut}_{\pi} Z_{0} \rightarrow \operatorname{Aut}_{\#}^{N} Z_{0}
$$

is an isomorphism.

Moreover if $H^{>M}(Z ; \mathbb{Z})=0$, then there is some integer $N$ such that $\operatorname{Aut}_{\pi} Z \cong \operatorname{Aut}_{\#}^{N} Z$.

Example 3. Suppose $W$ is the fat wedge of the three spheres $S^{2}, S^{2}$ and $S^{4}$, ie. $S^{2} \times S^{2} \times S^{4}=$ $W \cup_{\omega} D^{8}$ where $\omega \in \pi_{7}(W)$ denotes a triple Whitehead product. Then the rational homotopy Lie algebra of $W$ is generated by elements of degrees less than or equal to 6 :

$$
\pi_{*}(\Omega W) \otimes \mathbb{Q} \cong A b\left(x_{1}, y_{1}, z_{3}\right) \coprod \mathbb{L}\left(t_{6}\right)
$$


The cokernel of the morphism $\operatorname{Aut}_{\#}^{7}(W) \rightarrow \operatorname{Aut}_{\#}^{6}(W)$ is not finite, but for $n \geqslant 8$, the cokernel of the correponding injection $\operatorname{Aut}_{\#}^{n}(W) \rightarrow \operatorname{Aut}_{\#}^{n-1}(W)$ is finite.

Example 4. The integer $N$ depends on the space and not only on the dimension of the space. Consider for instance the 6 -dimensional rational space $X$ whose minimal Lie model is given by the following differential graded Lie algebra

$$
\begin{gathered}
L=\left(\mathbb{L}\left(x_{i}, y_{i}, z_{i}, a_{i, j}, b_{i, j}, u, v, w\right)_{i, j=1, \ldots, n ; i \leqslant j}, d\right) \\
\left|x_{i}\right|=\left|y_{i}\right|=\left|z_{i}\right|=2,|u|=1,|v|=2,\left|a_{i, j}\right|,\left|b_{i, j}\right|,|w|=5, \\
d(u)=d(v)=d\left(x_{i}\right)=d\left(y_{i}\right)=d\left(z_{i}\right)=0, d\left(a_{i, j}\right)=\left[x_{i}, z_{j}\right], d\left(b_{i, j}\right)=\left[y_{i}, z_{j}\right], \text { and } \\
d(w)=\left[x_{1}, y_{1}\right]+\ldots+\left[x_{n}, y_{n}\right] .
\end{gathered}
$$

The autoomorphism $\varphi$ equal to the identity on all generators except $w$ and defined on $w$ by $\varphi(w)=w+[v,[v, u]]$ belongs to $\operatorname{Aut}_{\#}^{5+2 n}(X)$ but not to $\operatorname{Aut}_{\pi}(X)$.

Proof of Theorem 3. Denote by $(\wedge V, d)$ the Sullivan minimal model of $Z([\mathbf{1 7}])$. We denote by Aut $(\wedge V, d)$ the group of homotopy classes of automorphisms of $(\wedge V, d)$. Each automorphism of $(\wedge V, d)$ induces an isomorphism on the vector space of indecomposable elements $Q(\wedge V)=$ $\wedge V / \wedge^{2} V \cong V$. We denote by $\operatorname{Aut}_{\pi}(\wedge V, d)$ the kernel of the representation morphism $\operatorname{Aut}(\wedge V, d) \rightarrow \operatorname{Aut}(Q(V))$ and by $\operatorname{Aut}_{\#}^{N}(\wedge V, d)$ the kernel of the morphism $\operatorname{Aut}(\wedge V, d) \rightarrow$ $\operatorname{Aut}\left(Q(V)^{\leqslant N}\right)$. We denote also by $I s o(\wedge V, d)$ the group of automorphisms of $(\wedge V, d)$, and by $I s o_{1}(\wedge V, d)$ the subgroup of autoomorphisms of the form $i d+\varphi$, with $\varphi: V \rightarrow \wedge^{\geqslant 2} V$.

From the Sullivan theory of minimal models one deduce the following group isomorphisms

$$
\left\{\begin{array}{l}
\operatorname{Aut}_{0}=\operatorname{Aut}(\wedge V, d) \\
\operatorname{Aut}_{\#}^{N} Z_{0}=\operatorname{Aut}_{\#}^{N}(\wedge V, d) \\
\operatorname{Aut}_{\pi} Z_{0}=\operatorname{Aut}_{\pi}(\wedge V, d)
\end{array}\right.
$$

where $Z_{0}$ denote the 0-localization of $Z$.

Since $H^{>M}(\wedge V, d)=0$, each automorphism of $\left(\wedge V^{\leqslant M}, d\right)$ extends to an automorphism of $(\wedge V, d)$. This means that the restriction map

$$
\rho: \operatorname{Aut}(\wedge V, d) \rightarrow \operatorname{Aut}\left(\wedge V^{\leqslant M}, d\right)
$$

is onto. Let $\theta:(\wedge V, d) \rightarrow(A, d)$ be a quasi-isomorphism with $A^{>M}=0$. Suppose now that $f, g \in \operatorname{Aut}(\wedge V)$. If $\rho(f) \sim \rho(g),(\sim$ means homotopy in the category of c.d.g.a.'s $)$, then $\theta \circ f \sim \theta \circ g$, and since $\theta$ is a quasi-isomorphism, $f \sim g$. This shows that $\rho$ is also injective.

We form the vector space $\operatorname{Hom}^{0}\left(V^{\leqslant M}, \wedge^{\geqslant 2} V\right)$, consisting of the degree zero linear maps. We choose an homogeneous basis, $\left(x_{i}\right)_{i=1, \ldots, N_{1}}$, of $V \leqslant M$, and an homogeneous basis, $\left(y_{j}\right)_{j=1, \ldots, N_{2}}$, for $\left(\wedge^{\geqslant 2} V\right)^{\leqslant M}$. Then for any $\varphi \in \operatorname{Hom}^{0}(V \leqslant M, \wedge \geqslant 2 V)$ we write

$$
\varphi\left(x_{i}\right)=\sum_{j} \alpha_{i}^{j} y_{j}
$$

Of course $i d+\varphi$ is an automorphism of $\left(\wedge V^{\leqslant M}, d\right)$ if and only if it commutes with the differential, i.e. if and only if, for $i=1, \ldots, N_{1}$, we have

$$
(i d+\varphi) d\left(x_{i}\right)=d\left(x_{i}+\varphi\left(x_{i}\right)\right) .
$$

By expressing these conditions in terms of the $\alpha_{i}^{j}$, we obtain a bijective correspondance between Iso $1\left(\wedge V^{\leqslant M}, d\right)$ and some sub-algebraic variety $W$ in $\mathbb{Q}^{N_{1} N_{2}}$.

We choose a linear section, $\sigma:\left(d\left(\wedge^{\geqslant 2} V\right)\right)^{>M} \rightarrow(\wedge V)^{\geqslant M}$, of the differential $d$. Then each $\psi=i d+\varphi$ in $I s o_{1}\left(\wedge V^{\leqslant M}, d\right)$ extends in a natural way to an automorphism of $(\wedge V, d)$ by the following rule : Let $z \in V^{M+k}, k \geqslant 1$, then $\psi d z$ is a coboundary so that we put $\psi(z)=\sigma \psi d z$. 
Performing this construction on a homogeneous basis of each $V^{n+k}$ we construct the required homomorphism. Moreover, $\psi$ is an isomorphism since it is a quasi-isomorphism and $(\wedge V, d)$ is minimal ([17]). We put $\Gamma_{M}=I s o_{1}\left(\wedge V^{\leqslant M}, d\right)$, and for $m \geqslant M$,

$$
\Gamma_{m}=\left\{\psi \in \Gamma, \psi(z)-z \in \wedge^{\geqslant 2} V, \quad \text { for } z \in V^{\leqslant m}\right\} .
$$

the choice of the section isomorphisms of $(\wedge V, d)$. We $\psi$ such that in their extension

Now the sequence

$$
\ldots \subset \Gamma_{M+2} \subset \Gamma_{M+1} \subset \Gamma_{M}
$$

is a sequence of inclusions of algebraic varieties. Since $\mathbb{Q}\left[\alpha_{i}^{j}\right]$ is noetherian, this sequence stabilizes : there is an integer $N$ such that

$$
\Gamma_{N}=\Gamma_{N+r} \quad \forall r \geqslant 1 .
$$

Denote by $\Gamma_{i d}$ the normal subgroup of $\Gamma$ consisting of the automorphisms homotopic to the identity. Then $f \sim g$ if and only if $f g^{-1} \in \Gamma_{i d}$. Thus Aut $\left(\wedge V^{\leqslant M}, d\right)$ is isomorphic to the quotient $\left.I s o_{1}\left(\wedge V^{\leqslant M}, d\right)\right) / \Gamma_{i d}$. This yields the equality

$$
\operatorname{Aut}_{\#}^{N}(\wedge V, d)=\operatorname{Aut}_{\#}^{N+r}(\wedge V, d) \quad \forall r \geqslant 1 .
$$

Since $\operatorname{Aut}_{\#}^{k}(\wedge V, d) \cong \operatorname{Aut}_{\#}^{k}\left(Z_{0}\right),([\mathbf{1 7}])$, we obtain finally

$$
\operatorname{Aut}_{\#}^{N} Z_{0}=\operatorname{Aut}_{\#}^{N+r} Z_{0} \quad \forall r \geqslant 1
$$

Suppose now that $Z$ is a simply connected finite CW complex. Then $\operatorname{Aut}_{\pi}(Z) \subset \operatorname{Aut}_{\#}^{N}(Z)$ is an normal inclusion of finitely generated nilpotent groups $([\mathbf{9}])$ with same Malcev completions. It is then easy to see by induction on the nilpotency index that the quotient is a finite group. Therefore there is an integer $P \geqslant N$ such that $\operatorname{Aut}_{\pi}(Z) \cong \operatorname{Aut}_{\#}^{P}(Z)$.

\section{Proof of Theorem 2}

The proof of Theorem 2 is based on a sequence of 4 lemmas.

Lemma 1. There is a rational homotopy equivalence $K: W \rightarrow X$ where $W$ is a finite $C W$ complex.

Henceforth we consider $K: W \rightarrow X$ as in lemma 1, and by Theorem 3, we fix an integer $N$ such that $\mathrm{Aut}_{\#}^{N} X_{0}=\operatorname{Aut}_{\pi} X_{0}$ and $H^{\geqslant N}(X ; \mathbb{Q})=0$.

Lemma 2. The morphism Aut $_{\pi} W^{(n+1)} \stackrel{f \rightarrow f^{(n)}}{\longrightarrow} \operatorname{Aut}_{\pi} W^{(n)}$ is injective and has a finite cokernel for each $n>N$.

Lemma 3. The morphism $\operatorname{Aut}_{\pi} X^{(n+1)} \stackrel{f \rightarrow f^{(n)}}{\longrightarrow} \operatorname{Aut}_{\pi} X^{(n)}$ has a finite kernel and a finite cokernel for $n \geqslant N$.

Theorem 2 follows from previous lemmas and the following lemma due to McGibbon and Möller ([12], Lemma 3).

Lemma 4. Let $G_{n} \stackrel{q_{n}}{\longrightarrow} G_{n-1}$ be a tower of groups. Suppose that $q_{n}\left(G_{n}\right)$ has finite index into $G_{n-1}$, for $n>N$. Then $\lim _{\leftarrow}^{1} G_{n}=\{*\}$ if and only if $\lim _{\leftarrow} G_{n} \rightarrow G_{N}$ has a finite cokernel.

\section{Proof of Lemma 1.}

Suppose that $H^{>M}(X ; \mathbb{Q})=0$. We consider the cellular chain complex of $X$,

$$
C_{M+1}(X ; \mathbb{Z}) \stackrel{\partial}{\rightarrow} C_{M}(X ; \mathbb{Z}) \rightarrow C_{M-1}(X ; \mathbb{Z}) \rightarrow \cdots
$$


where each $C_{k}(X ; \mathbb{Z})$ is freely generated by the $k$-cells of $X$ as a $\mathbb{Z}$-module. In particular, $C_{M+1}(X ; \mathbb{Z})=\oplus_{i=1}^{r} \mathbb{Z} e_{i}$. By an elementary argument in linear algebra, there exists elements $i_{1}, \cdots, i_{s} \in\{1, \cdots, r\}$ such that $\partial \otimes \mathbb{Q}$ sends isomorphically $\oplus_{j=1}^{s} \mathbb{Q} e_{i_{j}}$ to the image of $\partial \otimes \mathbb{Q}$ in $C_{M}(X ; \mathbb{Q})$.

Denote by $W$ the subcomplex of $X$ obtained by adding to the $M$-skeleton of $X$ the $(M+1)$ cells $e_{i_{1}}, \cdots, e_{i_{s}}$. The injection $K: W \rightarrow X$ induces an isomorphism in the homology of the rational cellular chain complexes and is therefore a rational homotopy equivalence.

Proof of Lemma 2. Since $n>N>\operatorname{dim} W$, and since $W^{(n)}=W \cup_{\varphi_{\alpha}} e^{n_{\alpha}}, n_{\alpha}>n+2$, the restriction maps

$$
\left\{\begin{array}{l}
\operatorname{Aut}_{\#}^{n} W^{(n+1)} \rightarrow \mathrm{Aut}_{\#}^{n} W^{(n)} \\
\operatorname{Aut} W \rightarrow \operatorname{Aut} W^{(n+1)} \rightarrow \operatorname{Aut} W^{(n)}
\end{array}\right.
$$

are isomorphisms. This gives directly the first part of the lemma.

Consider the following diagram obtained by restriction and rationalization

$$
\begin{array}{ccccc}
\mathrm{Aut}_{\#}^{n+1} W^{(n+1)} & \stackrel{\rho}{\hookrightarrow} & \mathrm{Aut}_{\#}^{n} W^{(n+1)} & \stackrel{\text { rep }}{\longrightarrow} & \operatorname{Aut} \pi_{n+1}(W) \\
r^{\prime} \downarrow & & \downarrow^{r} & & \\
\operatorname{Aut}_{\#}^{n+1} W_{0}^{(n+1)} & = & \operatorname{Aut}_{\#}^{n} W_{0}^{(n+1)} & \stackrel{r e p_{0}}{\longrightarrow} & \operatorname{Aut} \pi_{n+1}(W) \otimes \mathbb{Q}
\end{array}
$$

By hypothesis, (cf. Theorem 3), the representation map $r e p_{0}$ is trivial, the image of the representation rep : $\operatorname{Aut}_{\#}^{n} W^{(n+1)} \rightarrow$ Aut $\pi_{n+1}(W)$ is finite and thus its kernel $\operatorname{Aut}_{\#}^{n+1} W^{(n+1)}$ is a subgroup of finite index.

Therefore the composite

$$
\operatorname{Aut}_{\pi} W^{(n+1)}=\operatorname{Aut}_{\#}^{n+1} W^{(n+1)} \hookrightarrow \operatorname{Aut}_{\#}^{n} W^{(n+1)} \rightarrow \operatorname{Aut}_{\#}^{n} W^{(n)}=\operatorname{Aut}_{\pi} W^{(n)}
$$

has a finite cokernel.

Proof of Lemma 3. We use the same argument as in ([12]). We define

$$
\Delta\left(f^{(n)}\right)=\left\{(\alpha, \beta) \in \operatorname{Aut}_{\pi} W^{(n)} \times \operatorname{Aut}_{\pi} X^{(n)} \mid f^{(n)} \alpha=\beta f^{(n)}\right\}
$$

Then $\Delta\left(f^{(n)}\right)$ is a group and since $f: W \rightarrow X$ is a rational equivalence, by a result of Wilkerson ([19]) the projections from $\Delta\left(f^{(n)}\right)$ to $\operatorname{Aut}_{\pi} W^{(n)}$ and to $\operatorname{Aut}_{\pi} X^{(n)}$ have finite kernels and cokernels.

Now, using Lemma 2, a simple diagram chasing yields the result.

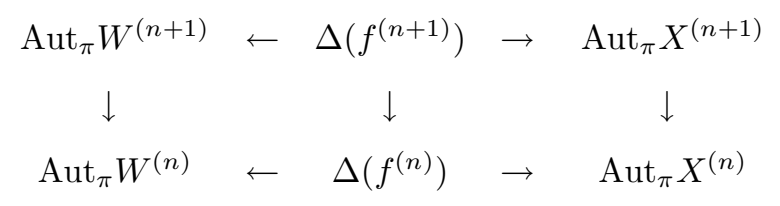

\section{Example of a space $X$ with $\operatorname{SNT}_{\pi}(X) \neq\{[X]\}$}

Consider the CW complex

$$
Z=\left(S_{a}^{2} \times S_{b}^{2} \times \bigvee_{p \geqslant 1} S^{6 p}\right) \vee\left(\bigvee_{n \geqslant 1} S_{n}^{4}\right) \cup_{\left(\gamma_{n, p}\right)} \bigcup_{\substack{p \geqslant 1 \\ n>p}} e_{n}^{4+6 p},
$$

where $\gamma_{n, p}$ is the Whitehead bracket $\left[S_{n}^{4}, S^{6 p}\right]$. 
The space $T$ is constructed as a deformation of the space $Z$.

$$
T=\left(\left(S_{a}^{2} \vee S_{b}^{2}\right) \times\left(\vee_{p \geqslant 1} S^{6 p}\right)\right) \cup_{\left[S_{a}^{2}, S_{b}^{2}\right]} e^{4} \vee\left(\bigvee_{n \geqslant 1} S_{n}^{4}\right) \cup_{\left(\gamma_{n, p}\right)} \bigcup_{\substack{p \geqslant 1 \\ n>p}} e_{n}^{4+6 p} \cup_{\bar{\omega}_{p}} \bigcup_{p \geqslant 1} e^{4+6 p},
$$

where $\bar{\omega}_{p}=\omega_{p}+\sum_{j=1}^{p}\left[S^{6 p}, S_{j}^{4}\right]$. Here the element $\omega_{p}$ is a triple Whitehead product in the $\pi_{6 p+3}$ of the fat wedge $T\left(S_{a}^{2}, S_{b}^{2}, S^{6 p}\right)$.

The spaces $X$ and $Y$ are respectively the rationalizations of $Z$ and $T$. We show that $X$ and $Y$ do not have the same homotopy type, and that there exists a sequence of homotopy equivalences between the $n^{\text {th }}$ skeleton

$$
f_{n}: X_{n} \rightarrow Y_{n}
$$

such that

$$
\pi_{r}\left(f_{n}\right)=\pi_{r}\left(f_{m}\right)
$$

when $r \leqslant n \leqslant m$. In particular $f_{n}$ induces an homotopy equivalence $f_{n}^{(n-1)}: X^{(n-1)} \rightarrow Y^{(n-1)}$, so that $\operatorname{SNT}_{\pi}(X) \neq\{X\}$.

By Quillen rational homotopy theory $([\mathbf{1 4}])$, there is an equivalence of homotopy category between the homotopy category of simply connected CW complexes and the homotopy category of connected differential graded Lie algebras. It thus suffices to prove the corresponding results into the category of differential graded Lie algebras.

The Quillen models $\mathcal{L}_{X}$ and $\mathcal{L}_{Y}$ of $X$ and $Y$ are respectively given by :

$$
\mathcal{L}_{X}=\left(\mathbb{L}\left(a, b, c, x_{i}, y_{i}, v_{i}, w_{i}, t_{i}, z_{i, j} ; \quad i \geqslant 1, j>i\right), d\right),
$$

with $|a|=|b|=1,|c|=3,\left|x_{i}\right|=6 i-1,\left|y_{i}\right|=3,\left|z_{i, j}\right|=6 i+3,\left|v_{i}\right|=6 i+1,\left|w_{i}\right|=6 i+1$, $\left|t_{i}\right|=6 i+3, d(c)=[a, b], d\left(x_{i}\right)=0, d\left(y_{i}\right)=0, d\left(z_{i, j}\right)=\left[x_{i}, y_{j}\right], d\left(v_{i}\right)=\left[a, x_{i}\right], d\left(w_{i}\right)=\left[b, x_{i}\right]$, $d\left(t_{i}\right)=\omega_{i}=\left[v_{i}, b\right]+\left[c, x_{i}\right]+\left[w_{i}, a\right]$.

$$
\mathcal{L}_{Y}=\left(\mathbb{L}\left(a, b, c, x_{i}, y_{i}, v_{i}, w_{i}, t_{i}, z_{i, j} ; \quad i \geqslant 1, j>i\right), d\right),
$$

with same degrees and differentials, except that

$$
d\left(t_{i}\right)=\omega_{i}+\sum_{j=1}^{i}\left[x_{i}, y_{j}\right]
$$

Suppose that $\varphi: \mathcal{L}_{X} \rightarrow \mathcal{L}_{Y}$ is a quasi-isomorphism and denote by $\mathbb{L}^{p}$ the ideal of $\mathcal{L}_{X}$ or $\mathcal{L}_{Y}$ generated by iterated brackets of lenght $\geqslant p$.

By composition with an automorphism of $\pi_{2}(X) \otimes \mathbb{Q}=a \mathbb{Q} \oplus b \mathbb{Q}$ we can suppose that $\varphi(a)=a$ and $\varphi(b)=b$. Then we have

$$
\left\{\begin{array}{l}
\varphi(c)=c+\sum_{k \geqslant 1} \gamma_{k} y_{k} \\
\varphi\left(x_{i}\right)=\mu x_{i} \quad \bmod \mathbb{L}^{2}, \mu \neq 0 \\
\varphi\left(v_{i}\right)=\mu v_{i} \quad \bmod \mathbb{L}^{2} \\
\varphi\left(w_{i}\right)=\mu w_{i} \quad \bmod \mathbb{L}^{2} \\
\varphi\left(t_{i}\right)=\delta t_{i}+\sum_{j>i} \beta_{i, j} z_{i, j}
\end{array}\right.
$$

and,

$$
(d \varphi-\varphi d)\left(t_{i}\right)=(\delta-\mu) \omega_{i}+\sum_{j=1}^{i} \delta\left[x_{i}, y_{j}\right]+\sum_{j>i} \beta_{i, j}\left[x_{i}, y_{j}\right]-\sum_{k \geqslant 1} \gamma_{k} \mu\left[y_{k}, x_{i}\right] \bmod \mathbb{L}^{3} .
$$


Therefore $\gamma_{k} \neq 0$ for $k \leqslant i$. Since this has to be true for any $i \geqslant 1$, we have

$$
\varphi(c)=c+\sum_{k \geqslant 1} \gamma_{k} y_{k}
$$

with all $\gamma_{k} \neq 0$, which is impossible.

On the other hand, by taking $\mu=\delta=\gamma_{k}=1$ for $k \leqslant p$, and $\gamma_{k}=0$ for $k>p$, we obtain an homotopy equivalence $f_{n}: X^{n} \rightarrow Y^{n}$ with $n=4+6 p$.

Remark now that the composition of injections

$$
\left(\left(S^{2} \times S^{2} \times\left(\vee_{p} \geqslant 1 S^{6 p}\right)\right) \vee\left(\vee_{n} \geqslant 1 S_{n}^{4}\right)\right)_{0} \stackrel{i}{\rightarrow} X \rightarrow\left(\left[\left(S^{2} \times S^{2}\right) \vee\left(\vee_{n \geqslant 1} S_{n}^{4}\right)\right] \times\left[\vee_{p} \geqslant 1 S^{6 p}\right]\right)_{0}
$$

induces a surjective map on homotopy groups. It results then from results of Anick ([2]) that $\pi_{*}(i)$ is a surjective map, and using this it is clear that $\pi_{r}\left(f_{m}\right)=\pi_{r}\left(f_{n}\right)$ when $r \leqslant m \leqslant n$.

Theorem 4. Let $X$ be the rationalizations of the $C W$ complex

$$
Z=\left(S_{a}^{2} \times S_{b}^{2} \times \bigvee_{p \geqslant 1} S^{6 p}\right) \vee\left(\bigvee_{n \geqslant 1} S_{n}^{4}\right) \cup_{\left(\gamma_{n, p}\right)} \bigcup_{\substack{p \geqslant 1 \\ n>p}} e_{n}^{4+6 p},
$$

where $\gamma_{n, p}$ is the Whitehead bracket $\left[S_{n}^{4}, S^{6 p}\right]$. Then

$$
\operatorname{SNT}_{\pi}(X) \cong \lim _{\leftarrow}{ }^{1} \operatorname{Aut}_{\pi} X^{(n)} \cong \prod_{n \geqslant 1} \mathbb{Q} / \bigoplus_{n \geqslant 1} \mathbb{Q}
$$

Proof. Since $\pi_{*}(\Omega X) \otimes \mathbb{Q}$ is generated as an algebra by the elements $a, b, x_{i}$ and $y_{i}, i \geqslant 1$, with the notations of section 4 , the restriction map Aut $X \rightarrow \underset{\leftarrow}{\lim } G_{n}$ is surjective. Therefore $\operatorname{SNT}_{\pi}(X)=\lim _{\leftarrow}^{1} \operatorname{Aut}_{\pi} X^{(n)}$.

Denote by $\mathcal{L}_{X}^{(n)}$ the quotient of $\mathcal{L}_{X}$ by the ideal $\mathcal{J}=\left(\mathcal{L}_{X}\right)^{>n+1} \oplus S$, where $S=d\left(\mathcal{L}_{X}^{n+2}\right)$. Clearly Aut ${ }_{\pi} X^{(n)}$ can be identified with the group of homotopy classes of automorphisms $\varphi$ of $\left.\mathcal{L}_{X}^{(n}\right)$ such that $\varphi$ is the identity on the vector space generated by the elements $a, b, x_{i}, y_{i}$, $i \geqslant 1$.

To each $\varphi \in$ Aut $_{\pi} X^{(n)}, n \geqslant 4$, we can associate the element $\varphi(c)-c \in \oplus_{i \geqslant 1} \mathbb{Q} y_{i}$. We define in this way a linear map Aut ${ }_{\pi} X^{(n)} \rightarrow \pi_{3}(\Omega X)$ whose kernel and cokernel are respectively denoted by $K_{n}$ and $I_{n}$;

$$
0 \rightarrow K_{n} \rightarrow \text { Aut }_{\pi} X^{(n)} \rightarrow I_{n} \rightarrow 0 .
$$

For $\varphi \in K_{n}$ and $n \geqslant 6 i+3$, the elements $\varphi\left(v_{i}\right)-v_{i}$ and $\varphi\left(w_{i}\right)-w_{i}$ belong to the sub Lie algebra $L$ generated by $a, b$ and the $x_{j}$. This define a map

$$
\psi: K_{n} \rightarrow \bigoplus_{i \mid 6 i+3 \leqslant n}\left(L_{6 i+3} \oplus L_{6 i+3}\right),
$$

and this gives a short exact sequence

$$
0 \rightarrow K_{n}^{\prime}=K e r \psi \rightarrow K_{n} \stackrel{\psi}{\longrightarrow} \bigoplus_{i \mid 6 i+3 \leqslant n}\left(L_{6 i+3} \oplus L_{6 i+3}\right) \rightarrow 0 .
$$

Since the induced map $\psi: \lim _{\leftarrow} K_{n} \rightarrow \bigoplus_{i \geqslant 1}\left(L_{6 i+3} \oplus L_{6 i+3}\right)$ is surjective, we have

$$
\lim _{\leftarrow}^{1} K_{n} \cong \lim _{\leftarrow}^{1} K_{n}^{\prime}
$$


Now remark that the sequence $K_{n}^{\prime} \rightarrow K_{n-1}^{\prime}$ is a sequence of surjective maps. It follows that $\lim { }^{1} K_{n}^{\prime}=0$. This gives the isomorphism

$$
\lim _{\leftarrow}^{1} \text { Aut }{ }_{\pi} X^{(n)} \cong \lim _{\leftarrow}^{1} I_{n}
$$

Now, clearly, $I_{6 i+4}=\bigoplus_{j>i} \mathbb{Q} y_{j}$. We consider then the short exact sequence of towers

$$
0 \rightarrow \bigoplus_{j \leqslant i} \mathbb{Q} y_{j} \rightarrow \bigoplus_{j \geqslant 1} \mathbb{Q} y_{j} \rightarrow \bigoplus_{j>i} \mathbb{Q} y_{j} \rightarrow 0
$$

The associated six-terms exact sequence reduces to the exact sequence

$$
0 \rightarrow \bigoplus_{j \geqslant 1} \mathbb{Q} y_{j} \rightarrow \prod_{j \geqslant 1} \mathbb{Q} y_{j} \rightarrow \lim _{\leftarrow}^{1} I_{n} \rightarrow 0
$$

\section{References}

1. J.F. Adams. An example in homotopy theory. Proc. Cambridge Philosophical Society 53 (1957), 922-923.

2. D. Anick. Non commutative graded algebras and their Hilbert series. J. of Algebra 78 (1982), 120-140.

3. M. Arkowitz and K.-I. Maruyama. Self homotopy equivalences which induce the identity on homology, cohomology or homotopy groups. Preprint.

4. A.K. Bousfield and D. Kan. Homotopy limits, completions and localizations. Lecture Notes in Mathematics 304, Springer-Verlag (1972).

5. E. Dror and A. Zabrodsky. Unipotency and nilpotency in homotopy equivalences. Topology 18 (1979), 187-197.

6. Y. Félix, S. Halperin and J.-C. Thomas. Elliptic spaces Bulletin of the Amer. Math. Soc. 25, (1991) 69-73.

7. B. Gray. Spaces of the same n-type, for all $n$. Topology 5 (1966), 241-243.

8. C. Jensen. Les foncteurs dérivés de lim et leurs applications en théorie des modules. Lecture Notes in Mathematics 254, Springer-Verlag, 1972.

9. K.-I. Maruyama. Finitely presented subgroups of self-homotopy equivalences group. Math. Z. 221 (1996), 537-548.

10. C .A. McGibbon and J.M. Moller. On spaces of the same $n$-type for all $n$. Topology 31 (1992), 177-201.

11. C.A. McGibbon and J.M. Moller. How can you tell two spaces apart when they have the same $n$-type for all $n$ ? Adams Memorial Symposium on Algebraic Topology, London Math. Soc. Lecture Note Series 175 (1992), 131-144.

12. C.A. McGibbon and J.M. Moller. On infinite dimensional spaces that are rationally equivalent to a bouquet of spheres. Proceeding of the Barcelona conferences (1998).

13. C.A. McGibbon and C. Wilkerson. Loop space of finite complexes at large primes. Proc. Amer. Math. Soc. 96 (1986), 698-702

14. D. Quillen. Rational homotopy theory. Annals of Math. 90 (1969), 205-295. 
15. J. Roitberg. Weak identities, phantom maps and $H$-spaces. Israel J. Math. 66 (1989), 319-329.

16. D. Stanley. On the Lusternik-Schnirelmann category of maps, Preprint (1998).

17. D. Sullivan. Infinitesimal computations in topology. Publ. IHES 47 (1977), 269-331.

18. C. Wilkerson. Classification of spaces of the same $n$-type for all $n$. Proceedings of the American Mathematical Society 80 (1976), 279-285.

19. C. Wilkerson. Applications of minimal simplicial groups. Topology. 15 (1976), 111-130.

This article may be accessed via WWW at http://www.rmi.acnet.ge/hha/ or by anonymous ftp at ftp://ftp.rmi.acnet.ge/pub/hha/volumes/1999/n10/n10.(dvi,ps,dvi.gz,ps.gz)

Yves Fèlix felix@agel.ucl.ac.be

Départment de Mathématiques

Chemin du Cyclotron 2

1348 Louvain-la-Neuve

Belgique

Jean-Claude Thomas jean-claude.thomas@univ-angers.fr

Université d'Angers

Faculté des Sciences

2bd Lavoisier, Cedex 01

France 